\title{
Multilevel lumbar spondylolysis: case report and literature review
}

\author{
E.G. Skryabin, M.A. Akselrov, I.A. Lebedev
}

Tyumen State Medical University, Tyumen, Russian Federation

\begin{abstract}
Introduction Diagnosis and treatment of vertebrogenic pain syndrome caused by multilevel lumbar spondylolysis is crucial for patients of different age groups. Objective To report a clinical case of diagnosis and treatment of a two-level lumbar spondylolysis in a 16-year-old male patient, and review the relevant literature. Material and methods We had an experience of treatment and follow-up of a 16-year-old athlete who presented with low back pain and was diagnosed with spondylolysis of the pars interarticularis at the L3 and L5 levels. Diagnostic workup included patient history, physical examination, diagnostic imaging (plain radiograph, computed tomography). Scientific publications from modern medical databases were used for literature review. A total of 32 relevant articles brought out between 1975 and 2019 were reviewed. Results The 16-year-old athlete was diagnosed with multilevel spondylolysis of lumbar spine based on patient history, physical examination data and diagnostic imaging of lumbar spine. No pathognomonic clinical findings were revealed. The diagnosis was confirmed with computed tomography. Conservative treatment was initiated for the patient and a long-termfollow-up was available. Literature review included epidemiologic data characterizing multilevel lumbar spondylolysis: the indicence, patients' gender and age. Major etiological factors, common localization of the pathology, the incidence of spondylolysis and spondylolisthesis, treatment strategy including surgical procedures are described. Discussion There is a small number of scientific publications describing different aspects of multilevel spondylolysis of the lumbar spine in the current medical literature. The diagnosis is often delayed for the reason. The natural history of pain in adolescent athletes sustaining the load placed on them in both training and competition can be helpful for timely diagnosis. Conclusion The implications of this clinical case report can be practical for accentuation of multilevel spondylolysis of the lumbar spine among Russian specialists, contribute to medical knowledge and extend to both early diagnosis and efficient management. Keywords: multilevel spondylolysis, case report, literature review
\end{abstract}

\section{BACKGROUND}

Low back pain is a relatively common presenting symptom in children and adolescents who seek medical care with trauma and orthopaedic service. Spondylolysis of the pars interarticularis is one of the most common cause of vertebrogenic pain syndrome in growing patients $[1,2]$. The condition is not common for adults to be diagnosed at several levels [3-5]. Spondylolytic defects involving multiple vertebral levels, on the other hand, are extremely rare [6-9]. No articles reporting multilevel lumbar spondylolysis in children and adolescents could be found in the Russian medical literature. Objective To report a clinical case of diagnosis and treatment of a twolevel lumbar spondylolysis in a 16-year-old male patient, and review the relevant literature.

\section{MATERIAL AND METHODS}

We had an experience of treatment and follow-up of a 16-year-old athlete who presented with low back pain and was diagnosed with right-sided L3 and bilateral L5 spondylolysis. Diagnostic workup included patient history, physical examination, diagnostic imaging (plain radiograph, computed tomography). The original literature search was conducted on key resources including PubMed, eLIBRARY, CYBERLENINKA, Cochrane Library and used for literature review. A total of 32 relevant articles brought out between 1975 and 2019 were reviewed. Literature searches included both Russian and English keywords: спондилолиз (spondylolysis), многоуровневый спондилолиз (multilevel spondylolysis).

With proper consent obtained from the parents, we report case of a 16-year-old male adolescent athlete Sh. who presented with low back pain for one year with radiation to nowhere. His pain graded 3-4 points (30-40 mm) on Visual Analogue Scale. No spine injury was reported. He attended the gym for the last 18 months to improve his fitness level and do a military style 
strength training program. The pain initially appeared during or after training and resolved after rest in a horizontal position and sleep at night without taking drugs. The patient was diagnosed with adolescent osteochondrosis of lumbar spine by neurologist based on radiographic evaluation performed 6 months after he started experience pain. Medications were administered. Patient's father also had spine pain radiating to the right leg. Physical examination showed a sthenic adolescent with athletic build, well aligned head, slighlty asymmetric shoulder girdle, smooth thoracic kyphosis, lumbar lordosis, asymmetric hemithorax, no vertebral extensor muscle defense, sufficient minimally painful range of motion at active flexion, lateral flexion and rotation, tenderness to palpation over the spinous processes, the sacral crest and the greater ischiadic foramen. Axial loads appeared to be pain free with the straight leg raise test showing negative result. Length and circumference of lower limbs' segments were equal, no contractures in the joints of lower limbs revealed. The feet were not flattened. No disorders of algetic and tactile sensitivity observed in the lower limbs. No claudication symptoms were noted at the gait. Neither radiographs nor description of the radiological status were available. CT scans of lumbar spine revealed bilateral L5 (Fig. 1, a, b) and right-sided L3 (Fig. 1, a) spondylolysis.

Based on patient history, physical examination and diagnostic imaging the patient was diagnosed with vertebral lumbodynia, multilevel (bilateral L5 and right-sided L3) spondylolysis of lumbar spine (ICD-10: M43.0). The findings were interpreted for the adolescent and his parents. The patient was recommended to 1) restrict axial loads avoiding strength training exercises done while standing and at flexion and trunk extension, in particular, and injuries to the spine; 2) use a brace to fix lumbar spine; 3) physical therapy procedures (paraffin, ozokerite applications, electrophoresis with $5 \%$ calcium chloride solution, EHF therapy); 4) medications (courses of vitamins, spasmolytic drugs, chondroprotectors, spinal injections when indicated); 5) muscle relaxation massage for lumbar spine; 6) follow-up visit with orthopaedic and trauma surgeon at 6 months.

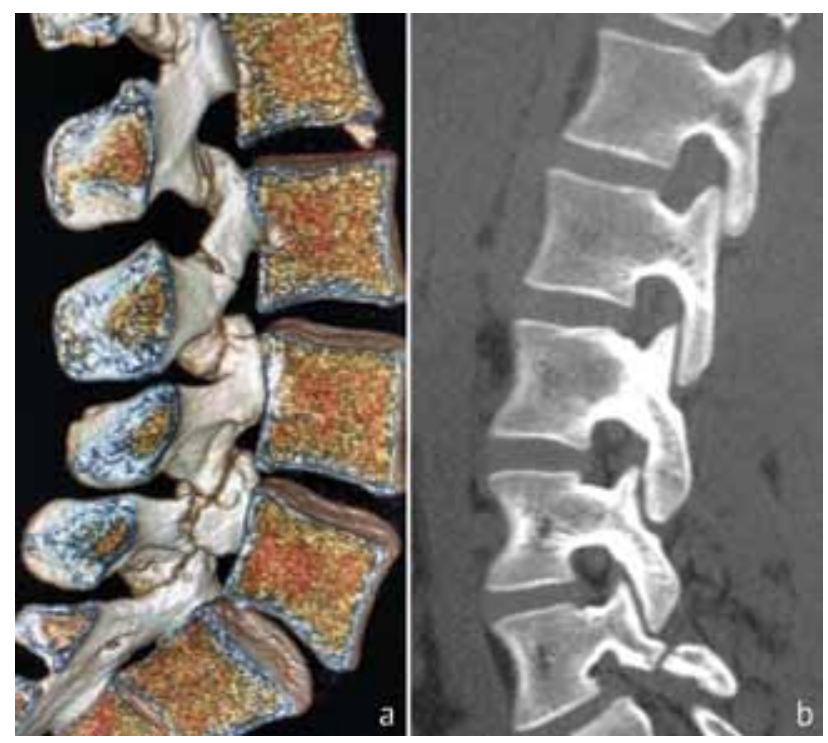

Fig. 1 CT scans of lumbar spine of the 16-year-old patient Sh. showing bilateral L5 $(\boldsymbol{a}, \boldsymbol{b})$ and right-sided L3 (a) spondylolysis

The patient was seen by an orthopaedist at $7 \frac{1}{2}$ months and was shown to be compliant with the treatment. He stopped doing strength training exercises and attended swimming pool twice a week. He demonstrated a pain free physical examination without deterioration in the local status of the spine. The patient was not evaluated radiologically. $\mathrm{He}$ was suggested continue swimming with lumbosacral movements at a small amplitude and avoid injuries to the lumbar and sacral spine. He was invited to attend the hospital for a follow-up visit at one year or earlier if he was likely to develop pain.

\section{RESULTS AND DISCUSSION}

Thirty two publications of foreign authors were identified through scrupulous literature search on the various aspects of the condition. The earliest article dates back to 1975 [10], and one of recent publications on the problem has been brought out in 2018 [3]. Case series were described in 26 articles with $18(69.23 \%)$ presenting one case report [3, 4, 7, 9-23]. Three cases of multilevel spondylolysis were reported in two articles $(7.73$ $\%)[6,8]$. The pathology was detected in $4,6,7$, 9, 10 and 13 patients and described in six articles [16, 24-27]. From 26 articles, one publication 
(3.84\%) presented the condition diagnosed at the cervical spine, left and right pars of $\mathrm{C} 4$ and C5 vertebrae [20]. The remaining 25 (96.16\%) described multilevel spondylolysis detected in lumbar spine. The incidence of the disease was reported in two articles only. G. Ravichandran [16] reported the incidence of multiple lumbar spondylolyses varying between $1.2 \%$ and 5.6 $\%$ but did not specify whether the incidence was reported among general population or in patients with confirmed spondylolyses. R. Molinari et al. [22] found a $5.6 \%$ incidence of multilevel spondylolysis in isolated cases (without associated spondylolisthesis).

Although the pathogenesis of lumbar spondylolysis remains controversial, it is widely accepted that spondylolysis has a genetic predisposition to the pars defect $[8,10,11]$ with higher incidence of spondylolysis in athletes and those participating in strenuous exercise [4, 7, 27]. Computed tomography is the best procedure for clearly visualizing spondylolysis when a pars fracture is present whereas magnetic resonance imaging is not commonly used for the diagnosis $[4,19,26,28]$. The pathology was detected at two levels in the majority of cases $(n=41$, $54.68 \%$ ). Three vertebrae were involved in 28 $(37.32 \%)$ individuals. Four patients $(5.33 \%)$ had spondylolysis at four levels [15, 18, 22, 30]. The pars defect was detected in 6 vertebrae in one patient $(1.33 \%)$ [14]. One publication (1.33\%) did not specify the number of vertebrae involved [9]. Specifically involved vertebrae were reported in twenty publications [3-8, 10-22, 26, 27]. The authors of the articles detected total 62 involved vertebrae. L4 was the commonly involved vertebra in $18(29.06 \%)$ cases. L3 and L5 appeared to be involved in $17(27.41 \%)$ and 15 (24.19\%) cases, respectively. L2 and L1 were found to be involved in $7(11.29 \%)$ and $2(3.22 \%)$ cases, respectively. $\mathrm{C} 4, \mathrm{C} 5$ and $\mathrm{T} 12$ were involved in one case each $(1.61 \%)$. Gender of the patients suffering from multilevel spondylolysis was reported in 18 articles [3, 4, 7, 9, 12-22, 25, 27, 30]. Summarizing the above details we found out that from 39 patients suffering from multilevel spondylolysis the pathology was detected in $28(71.79 \%)$ males and $11(28.21 \%)$ females. Thirteen articles [4, 7,
$9,12-15,18-22,30]$ reported the age at the initial presentation. The average age of patients when the pathology was first diagnosed was $351 / 2$ years. Only one 15-year-old female patient was under 18, the remaining patients aged from 19 to 60 years.

Three groups of authors $[19,21,22]$ reported cases of associated spondylolisthesis of lumbar spine in patients suffering from multilevel spondylolysis. Molinari R. et al. [22] reported a grade I spondylolisthesis at L5 level in a 32-yearold man diagnosed with L2, L3, L4 and L5 spondylolysis. Dunn A.S. et al. [19] reported a rare case of a grade I spondylolisthesis of L3 and a grade II spondylolisthesis of L4 secondary to bilateral pars interarticularis defects in a 43-yearold patient. Al Sebai M.W. et al. [21] described an unusual case of a combination of multiple bilateral spondylolyses of L2, L3, L4, sacralization of L5 and spondyloptosis at L4/5 in a 15-year-old female. The patient was treated surgically by posterior decompression, corpectomy and fusion in a three-stage operation with the follow-up of 2 years with no complications.

A variety of techniques can be used for treatment of multilevel spondylolysis. Treatment options include nonsurgical and operative methods including different fusion techniques. Literature review showed that treatment strategy was discussed in $21(67.74 \%)$ articles out of 32 . W.J. e Kim t al. [14], L.C. Wong et al. [9] and A.S. Dunn et al. [19] reported conservative treatment of the condition to include medications, bracing and physical therapy procedures. A.S. Dunn et al. [19] considered conservative management to be the standard of care for spondylolysis even in cases complicated with spondylolisthesis below grade III. If the results of non-operative methods proved unsuccessful J.H. Chang et al. [24] and D. Eingorn et al. [13] advocated use of numerous surgical techniques.

Patients with symptoms that persist for more than 6 months despite restriction of activity and bracing are candidates for surgery $[13,24]$. It is reported to be required in cases of established diagnosis of multilevel spondylolysis with low back pain [3-7, 12, 15-18, 20-22, 25-27, 30]. G. Sharifi et al. [17], J.H. Chang et al. [24] advise confirmation of the pars defect as the pain 
generator by means of a simple image-guided diagnostic anesthetic block of the pars prior to recommending surgical intervention. The most common procedure for surgical treatment of lumbar spondylolysis is spinal arthrodesis with/ without instrumentation combined with bone grafting and discectomy, if needed. J.H. Chang et al. [24] used the translaminar interfragmentary screw with bone grafting in 6 cases, and $\mathrm{H}$. Ogawa et al. [25] employed the wiring technique with bone grafting for the direct repair of the pars. B. Peng et al. [6] used compression screws for three-level spondylolysis in 3 cases. J.H. Chung et al. [26] reported direct repair of the pars interarticularis defects in six patients with multiple level spondylolysis with hook screws, translaminar screws and bone grafts. G. Sharifi et al. [17] reported favorable results with the hook-screw technique used to treat multilevel lumbar spondylolysis. M.R. Voisin et al. [3] presented a 50-year-old male patient with bilateral spondylolysis at L3 and L5 with associated spondylolisthesis who underwent the "smiley face" technique of direct multilevel repair without fusion using 3-dimensional intraoperative spinal navigation. K.H. Park et al. [15], D. Eingorn et al. [13] used an iliac crest autograft for direct repair of multilevel lumbar spondylolysis.

Direct repair can be performed via posterior and combined anterior and posterior approaches [4, 18]. According to Darnis et al. [4], B. Peng et al. [6], the preoperative factors as the state of intervertebral discs adjacent to the pars defects and patient's age are important for treatment strategy. In a young patient, extensive arthrodesis should be avoided, particularly if the discs are normal. In G. Ravichandran's opinion [16], when surgery is indicated, it must be radical, and the fusion should include all the defects and the intervening normal vertebrae and the sacrum. According to J. Mo et al. [31], spinal surgeons commonly choose to fix all the segments with pars interarticularis defect even those without slippage and not responsible for clinical symptoms in multi-level spondylolysis patients. T. Arai et al. [12] used the strategy for direct repair of 3-level lumbar spondylolysis in a 45-year-old man conducted for all three levels. There is a report of a 4-level lumbar spondylolysis in a 48-year-old female treated with respect to clinical symptoms at each of the levels including surgery for three levels and nonsurgical treatment performed for L5 pars lysis [30]. The reported results of surgical treatment performed for multilevel spondylolysis were shown to be mostly satisfactory for both the patients and the surgeons. A. Darnis et al. [4] reviewed the literature of the surgical management choices for 24 patients with multilevel spondylolysis reported by nine groups of authors $[7,12,13,15,16,21$, 24-26]. Long-term clinical and radiological outcomes were rates as 'excellent', 'good', 'fair' and 'poor'. The results of surgeries were evaluated as 'excellent' in $12(50.0 \%)$ cases out of 24 , as 'good' in $8(33.3 \%)$ patients. There were 2 $(8.33 \%)$ 'fair' and $2(8.33 \%)$ 'poor' outcomes. Excellent and good outcomes at a long term meant absence of vertebrogenic pain symptom, sufficient lumbar function, bone healing of the pars interarticularis and adequate instrumented fixation. Fair outcomes included persistent pain with physical activity and dissociation of construct components that could result in metallic sound with twisted trunk and removal of a system [12]. G. Ravichandran [16] reported poor results and complications of intertransverse fusion. According to B. Peng et al. [6], T. McKenna et al. [29] the success of management of the patients with multilevel spondylolyses depends on the choice of appropriate treatment for every patient. A preventative long-segment surgery was not considered to be necessary for multilevel spondylolysis as long as there were no soft tissue degeneration signs at adjacent level and it could cause significant biomechanical change at adjacent segment with pars defect [31].

The clinical case of the 16-year-old patient with low back pain presented is a common scenario when a young patient can be diagnosed with juvenile osteochondrosis of spine by a neurologist, family doctor, osteopathist, chiropractor with little effort. If a physical examination had revealed waist triangular asymmetry, asymmetrical paraspinal muscles, arch-shaped spinous processes deviation the patient would have been diagnosed with 
scoliosis. The two nosological entities of juvenile vertebrogenic pathology: juvenile osteochondrosis of spine (ICD-10: M42.0) and scoliosis (ICD-10: M41.0) are commonly diagnosed. Radiological evaluation can be useful to identify the condition with symptoms from the spinal motion segments determined as manifestations of juvenile osteochondrosis or scoliosis. Vertebrogenic pain syndrome at the lumbar spine has not been shown to be caused by the above conditions. Pains in children and adolescents can be caused by vertebral dysplasia and malformations that can have multiple patterns with delayed diagnosis. Spondylolysis of two lumbar vertebrae was also diagnosed with a delay of more than 6 months in the adolescent Sh. E. Nielsen et al. [32] quantified the time to diagnosis of spondylolysis/spondylolisthesis in young patients. Time from initial presentation to diagnosis was 25 weeks for nonorthopedic providers and 1 week for orthopedic surgeons. So, children suffering from back pain from spondylolysis may benefit from early referral to an orthopedic surgeon who will monitor the condition. In the case presented, time from initial presentation to diagnosis was 6 months (24-25 weeks) that is in line with the time reported by E. Nielsen et al. [32] with accuracy to one week.
One of unrecognized factors that would be helpful in timely diagnosis in our case can be the nature of the pain underevaluated in patient Sh. during and after strength training exercises. Strength training exercises suggest excessive stress on lumbar spine while tilting the body forward and backward. Hyperextension of lumbar spine is known to put the excessive stress on the pars interarticularis of the supersacral vertabra and can lead to pathological bone restructuring - spondylolysis - with resultant pain at the lumbar spine [1,2]. Strength exercises performed with hyperextended lumbar spine by an adolescent athlete with spondylolysis of the lower lumbar vertebrae developed intrauterine appear to result in pain caused by mechanical irritation of spondylolytic areas by lower articular processes of overlying vertebra [29]. Imaging of the lumbar spine performed with computed tomography would demonstrate the leading factor in the pathogenesis of pain with spondylolysis being most common [22, 26]. Computed tomography scan revealed L3 and L5 pars defects in the case presented. Medical recommendations of axial load restrictions for hyperextended lumbar spine, decompression methods including swimming provided pain relief in patient $\mathrm{Sh}$. for a long time.

\section{CONCLUSION}

Vertebrogenic pain syndrome caused by multilevel lumbar spondylolysis is crucial for patients of different age groups and not properly explored. Our scrupulous search of recent Russian medical literature on the problem provided no results. There was only a case of a 24 -year-old male suffering from mucopolysacharidosis type VI or Maroteaux-Lamy syndrome diagnosed with L3 and L4 pars defects described in the thesis of P.V. Ochirova 'Surgical treatment of patients with vertebral syndromes secondary to genetic disorders' (Russian Ilizarov Scientific Center for Restorative Traumatology and Orthopaedics, Kurgan, 2016). And only few cases are described in the existing foreign literature. Even the known types of isolated spondylolysis of the pars interarticularis are often in the sidelines of a more severe condition, spondylolisthesis, the primary focus of concerned specialists at diagnosis and treatment. Nearly half of patients with spondylolysis used to develop to spondylolisthesis and pars interarticularis defects are to be considered as the risk of progression to spondylolisthesis in young patients with developing spines $[5,13,22]$. The implications of this clinical case report can be practical for accentuation of multilevel spondylolysis of the lumbar spine among Russian specialists, contribute to medical knowledge and extend to both early diagnosis and efficient management. 


\section{REFERENCES}

1. Lawrence K.J., Elser T., Stromberg R. Lumbar spondylolysis in the adolescent athlete. Phys. Ther. Sport., 2016, vol. 20, pp. 56-60. DOI: 10.1016/j.ptsp.2016.04.003

2. Kessous E., Borsinger T., Rahman A., d'Hemecourt P.A. Contralateral Spondylolysis and Fracture of the Lumbar Pedicle in a Young Athlete. Spine, 2017, vol. 42, no. 18, pp. E1087-E1091. DOI: 10.1097/BRS.00000000000002086

3. Voisin M.R., Witiw C.D., Deorajh R., Guha D., Oremakinde A., Wang S., Yang V. Multilevel Spondylolysis Repair Using the «Smiley Face» Technique with 3-Dimensional Intraoperative Spinal Navigation. World Neurosurg., 2018, vol. 109, pp. e609-e614. DOI: 10.1016/j.wneu.2017.10.046

4. Darnis A., Launay O., Perrin G., Barrey C. Surgical management of multilevel lumbar spondylolysis: a case report and review of the literature. Orthop. Traumatol. Surg. Res., 2014, vol. 100, no. 3, pp. 347-351. DOI: 10.1016/j.otsr.2013.12.021

5. Zhang S., Ye C., Lai Q., Yu X., Liu X., Nie T., Zhan H., Dai M., Zhang B. Double-level lumbar spondylolysis and spondylolisthesis: A retrospective study. J. Orthop. Surg. Res., 2018, vol. 13, no. 1, pp. 55. DOI: 10.1186/s13018-018-0723-3

6. Peng B., Li D., Pang X. Surgical Management of 3-Level Lumbar Spondylolyses. Medicine (Baltomore), 2015, vol. 94, no. 27, pp. e1127. DOI: 10.1097/MD.0000000000001127

7. Hersh D.S., Kim Y.H., Razi A. Multi-level spondylolysis. Bull. NYU Hosp. Jt. Dis., 2011, vol. 69, no. 4, pp. 339-343.

8. Sairyo K., Sakai T., Yasui N., Kiapour A., Biyani A., Ebraheim N., Goel V.K. Newly occurred L4 spondylolysis in the lumbar spine with pre-existence L5 spondylolysis among sports players: case reports and biomechanical analysis. Arch. Orthop. Trauma Surg., 2009, vol. 129, no. 10, pp. 1433-1439. DOI: 10.1007/s00402-008-0795-3

9. Wong L.C. Rehabilitation of a patient with a rare multi-level isthmic spondylolisthesis: a case report. J. Can. Chiropr. Assoc., 2004, vol. 48, no. 2, pp. 142-151.

10.Privett J.T., Middlemiss J.H. Multiple lower lumbar spondylolyses. Br. J. Radiol., 1975, vol. 48, no. 574, pp. 866-869. DOI: 10.1259/0007-1285-48-574-866

11.Sakai T., Goda Y., Tezuka F., Abe M., Yamashita K., Takata Y., Higashino K., Nagamachi A., Sairyo K. Clinical features of patients with pars defects identified in adulthood. Eur. J. Orthop. Surg. Traumatol., 2016, vol. 26, no. 3, pp. 259-262. DOI: 10.1007/s00590015-1727-x

12.Arai T., Sairyo K., Shibuya I., Kato K., Dezawa A. Multilevel direct repair surgery for three-level lumbar spondylolysis. Case Rep. Orthop., 2013, vol. 2013, pp. 472968. DOI: 10.1155/2013/472968

13.Eingorn D., Pizzutillo P.D. Pars interarticularis fusion of multiple levels of lumbar spondylolysis. A case report. Spine, 1985, vol. 10, no. 3 , pp. 250-252.

14.Kim W.J., Song Y.D., Choy W.S. Multilevel Thoracolumbar Spondylolysis with Spondylolisthesis at L4 on L5. Clin. Orthop. Surg., 2015, vol. 7, no. 3, pp. 410-413. DOI: 10.4955/cios.2015.7.3.410

15.Park K.H., Ha J.W., Kim H.S., Moon E.S., Moon S.H., Lee H.M., Kim H.J., Kim J.Y. Multiple levels of lumbar spondylolysis - a case report. Asian Spine J., 2009, vol. 3, no. 1, pp. 35-38. DOI: 10.4184/asj.2009.3.1.35

16.Ravichandran G. Multiple lumbar spondylolyses. Spine, 1980, vol. 5, no. 6, pp. 552-557. DOI: 10.1097/00007632-19801100000011

17.Scharifi G., Jahanbakhshi A., Daneshpajouh B., Rahimzadeh A. Bilateral three-level lumbar spondylolysis repaired by hook-screw technique. Global Spine J., 2012, vol. 2, no. 1, pp. 51-56. DOI: 10.1055/s-0032-1307255

18.Schmid T., Heini P., Benneker L. A rare case of non-traumatic, multi-level, bilateral pedicle fractures of the lumbar spine in a 60-year-old patient. Eur. Spine J., 2017, vol. 26, no. Suppl.1, pp. 197-201. DOI: 10.1007/s00586-017-5-29-3

19.Dunn A.S., Baylis S., Ryan D. Chiropractic management of mechanical low back pain secondary to multiple-level lumbar spondylolysis with spondylolisthesis in a United States Marine Corps veteran: a case report. J. Chiropr. Med., 2009, vol. 8, no. 3, pp. 125-130. DOI: 10.1-16/j.jcm.2009.04.003

20. Yamamoto N., Miki T., Nasu Y., Nishiyama A., Dan'ura T., Matsui Y., Ozaki T. Congenital double-level cervical spondylolysis: a case report and review of the literature. Eur. Spine J., 2017, vol. 26, no. Suppl. 1, pp. 181-185. DOI: 10.1007/s00586-017-5005-y

21.Al-Sebai M.W., Al-Khawashki H. Spondyloptosis and multiple-level spondylolysis. Eur. Spine J., 1999, vol. 8, no. 1, pp. 75-77. DOI: $10.1007 / \mathrm{s} 005860050130$

22.Molinari R.W., McAssey R.N., Molinari A. Chronic Back Pain from Four-Level Lumbar Spondylolysis with Associated Spondylolisthesis: Case Report and Review of the Literature. Int. J. Neurorehabilitation, 2015, vol. 2, pp. 1000165. DOI: $10.4172 / 2376-0281.1000165$

23.Koné N., Soumaré O. Bilateral three-level lumbar spondylolysis. Pan. Afr. Med. J., 2017, vol. 27, pp. 105. DOI: 10.11604/ pamj.2017.27.105.12235

24.Chang J.H., Lee C.H., Wu S.S., Lin L.C. Management of multiple level spondylolysis of the lumbar spine in young males: a report of six cases. J. Formos. Med. Assoc., 2001, vol. 100, no. 7, pp. 497-502.

25.Ogawa H., Nishimoto H., Hosoe H., Suzuki N., Kanamori Y., Shimizu K. Clinical outcome after segmental wire fixation and bone grafting for repair of the defects in multiple level lumbar spondylolysis. J. Spinal Disord. Tech., 2007, vol. 20, no. 7, pp. 521-525. DOI: 10.1097/BSD.0b013e3180335c1f

26.Chung C.H., Chiu H.M., Wang S.J., Hsu S.Y., Wei Y.S. Direct repair of multiple levels lumbar spondylolysis by pedicle screw laminar hook and bone grafting: clinical, CT, and MRI-assessed study. J. Spinal Disord. Tech., 2007, vol. 20, no. 5, pp. $399-402$. DOI: 10.1097/01.bsd.0000211253.67576.90

27.Liu X., Wang L., Yuan S., Tian Y., Zheng Y., Li J. Multiple-level lumbar spondylolysis and spondylolisthesis. J. Neurosurg. Spine, 2015, vol. 22, no. 3, pp. 283-287. DOI: 10.3171/2014.10.SPINE14415

28.Goda Y., Sakai T., Sakamaki T., Takata Y., Higashino K., Sairyo K. Analysis of MRI signal changes in the adjacent pedicle of adolescent patients with fresh lumbar spondylolysis. Eur. Spine J., 2014, vol. 23, no. 9, pp. 1892-1895. DOI: 10.1007/s00586-0133109-6

29.McKenna T., Prideaux C.C. Multilevel Spondylolysis in an Adolescent Athlete: A Case Report. PM R, 2016, vol. 8, no. 9S, pp. S223. DOI: $10.1016 /$ j.pmrj.2016.07.228

30.Vidyadhara S. Rationale in the management of 4-level lumbar spondylolyses with or without instability and/or spondylolisthesis. Spine, 2014, vol. 39, no. 12, pp. E734-E738. DOI: 10.1097/BRS.0000000000000324 
Benij Ortopedii, Vol. 26, no 4, 2020

31.Mo J., Zhang W., Zhong D., Xu H., Wang L., Yu J., Luo Z. Is preventative long-segment surgery for multi-level spondylolysis necessary? A finite element analysis study. PLoS One, 2016, vol. 11, no. 2, pp. e0149707. DOI: 10.1371/journal.pone.0149707

32.Nielsen E., Andras L.M., Skaggs D.L. Diagnosis of spondylolysis and spondylolisthesis is delayed six months after seeing nonorthopedic providers. Spine Deform., 2018, vol. 6, no. 3, pp. 263-266. DOI: 10.1016/j.jspd.2017.10.008

Received: 06.11.2019

\section{Information about the authors:}

1.Evgeny G. Skryabin, M.D., Ph.D., Professor,

Tyumen state medical university, Tyumen, Russian Federation, ORCID:http://orcid.org/0000-0002-4128-6127, SPIN:4125-9422, Scopus ID:6507261198, Email: skryabineg@mail.ru

2.Mikhail A. Akselrov, M.D., Ph.D., Professor, Tyumen state medical university, Tyumen, Russian Federation, ORCID: http://orcid.org/0000-0001-6814-8894, SPIN:3127-9804, Email: akselrov@mail.ru

3. Ilya A. Lebedev, M.D., Ph.D., Professor, Tyumen state medical university, Tyumen, Russian Federation, ORCID: http://orcid.org/0000-0001-5405-7182,

Email: lebedef@inbox.ru 